\title{
ZUBIRI Y LA FILOSOFIA DE LA RELIGIÓN
}

\author{
ARMANDO SAVIGNANO \\ Università di Trieste
}

\begin{abstract}
RESUMEN: El artículo aborda la filosofía de la religión desde una confrontación crítica entre el punto de vista teorético de Zubiri, que está basado en la teoría de la inteligencia sentiente, y los métodos fenomenológico y hermenéutico. El autor señala la triple dimensión de la experiencia de Dios (individual, social e histórica) y el doble significado del concepto de realidad (como alteridad y como fundamento). La realidad como fundamento conduce al autor a enfrentar el concepto de religación, y la religación es, al mismo tiempo, una experiencia, un enigma y una manifestación del poder de la realidad. En este punto, el autor asevera que el hombre, que es una realidad relativamente absoluta, es una experiencia de Dios, que es una realidad absolutamente absoluta. En este contexto sale a luz la diferencia entre una investigación teológica y la dimensión teologal.
\end{abstract}

PALABRAS CLAVE: Dios, religación, experiencia, realidad, fundamento.

\section{Zubiri and Philosophy of Religion}

ABSTRACT: The article focuses on philosophy of religion starting from a critical comparison between Zubiri's theoretical point of view, which is based on sentient intelligence's theory, and phenomenological and hermeneutic methods. The author highlights the triple dimension of the experience of God (individual, social and historical) and the double meaning of the concept of reality (as otherness and as foundation). The reality as foundation brings the author to face the concept of re-ligation, and re-ligation is, at the same time, an experience, an enigma and a manifestation of the power of reality. At this stage, the author argues that man, who is a relatively absolute reality, is an experience of God, who is an absolutely absolute reality. In this context comes up the difference between a theological research and theologal dimension.

KEY WORDS: God, re-ligation, experience, reality, foundation.

De acuerdo con A. González ${ }^{1}$, la filosofía de la religión de Zubiri tiene rasgos de originalidad en comparación con los enfoques de la fenomenología religiosa, de la filosofía analítica y de la teología natural clásica. La actitud zubiriana implica una teoría de la inteligencia sentiente, de la que desarrolla una filosofía de la religión basada en la teoría de la re-ligación. La peculiaridad de la posición de Zubiri es el método filosófico que se centra en la teoría de la inteligencia sentiente, que también se aplica a la filosofía de la religión, en contraposición al método fenomenológico-hermenéutico, al análisis lingüístico y al enfoque crítico-explicativo a través de los cuales se estudia la religión. Esto no excluye los aportes antes mencionados, sino que los incluye superándolos a la luz de una perspectiva metodológica original y sistemática con la que Zubiri se acerca a la religión, especialmente en la trilogía dedicada al problema teologal del hombre. Tanto el análisis de las experiencias religiosas (la fenomenología de las

1 González, A. (2004), «Aproximación a la filosofía zubiriana de la religión», en Nicolás, J. A., Barroso, Ó. (eds.) (2004), Balance y perspectivas de la filosofía de X. Zubiri, Granada: Comares, pp. 265-282. 
religiones) como el análisis del lenguaje religioso (filosofías analíticas), se pueden emplear en un análisis del logos religioso (ver Zubiri, Inteligencia y logos), mientras que las teorías crítico-explicativas de la religión pueden complementarse con el análisis zubiriano de la razón religiosa (ver Inteligencia y razón ).

En otras épocas trataron de acercarse a Dios a través de la teología natural ${ }^{2}$, utilizando el orden del universo o el principio de causalidad: las pruebas cosmológicas. Alternativamente, el racionalismo moderno se basa en la Teodicea, subrayando la importancia y el papel del famoso argumento ontológico. Pero la razón, sola, no parece ser capaz de llegar directamente a Dios ni a través de los juicios analíticos, ni a través de los sintéticos. El hombre no puede llegar a Dios si Dios no se manifiesta a él de alguna manera. Por eso Dios no se halla sólo a través de la razón, sino sobre todo a través de la experiencia. Para Zubiri, la experiencia de Dios es individual, social e histórica. Cada vida humana es una experiencia de Dios, tal como se desprende del primer libro de la trilogía

2 El enfoque de Zubiri en torno a la teología natural no parece estar sujeto a las críticas de los protestantes, por ejemplo, la analogia entis de K. Barth en nombre de la concepción de Dios como «totalmente otro». Pero Zubiri insiste en la necesidad de liberarse de los conceptos griegos de acceso a Dios [ZuBiri, X. (1984), El hombre y Dios. Madrid: Alianza, p. 152]. Hay una cierta analogía con la posición de Brunner, E. (1963²), Das Problem des Naturrechtes. Zürich: Zwingli-Verlag, acerca de una concepción de la verdad como encuentro [véase ZuBIRI, X. (20012), Inteligencia y razón. Madrid: Alianza, pp. 258-263, 292-297]. Además, Zubiri, en la estela de algunas ideas neoplatónicas y de la misma teología negativa, no atribuye en primer lugar a Dios el concepto de ser, sino el concepto de realidad, que es una formalidad constitutivamente abierta, y también es alteridad, pero no en el sentido de las filosofías de la alteridad, que se relaciona con otros sujetos, sino una alteridad que se refiere a sí misma «por sí misma». Así se puede acercar la concepción zubiriana de Dios como realidad absoluta al concepto de totalmente otro de Barth. La analogía, para Zubiri, no es la ruta de acceso del mundo a Dios, sino un camino que desciende de Dios, que es la realidad absolutamente absoluta. Esta es la «analogía de lo absoluto», comparable con lo que el segundo Barth llama «analogía de la fe», que significa sacar a luz la humanidad de Dios. La analogía, para Zubiri, no afecta a la forma en que las personas pueden acceder a la divinidad, sino a la forma en que la deidad viene a los hombres. Los rasgos de unidad, personalidad e inteligencia pertenecen al hombre, que es relativamente absoluto (El hombre y Dios, p. 172). Sin embargo, hay una diferencia entre Zubiri, que sostiene estos argumentos desde el punto de vista filosófico, y Barth, que, en cambio, asume el dato revelado. La actitud de Zubiri puede ser fecunda también para purificar ciertos supuestos filosóficos implícitos en las mismas posiciones de Barth -que tiene una concepción casi hegeliana de la divinidad entendida como sujeto absoluto (cf. las críticas a Barth en Moltmann, J. (1980), Trinität und Reich Gottes. München: Kaiser, pp. 154-161) - y de Jungel, que concibe el dolor, el sufrimiento, la muerte a la manera griega de la negatividad. Así, la identificación de Dios con Cristo sería, para Jungel, concebir la negatividad en el seno mismo de la Divinidad. En cambio, para Zubiri, la encarnación de Dios no une a Dios con la nada, sino con las realidades positivas de la historia humana, aunque algunas de ellas son negativas desde un punto de vista ético. El mal no es pura nada, sino un estado real de las cosas [ZuBiri, X. (1992), Sobre el sentimiento y la volición. Madrid: Alianza, pp. 201-256], que Cristo enfrenta en la Cruz. Por lo tanto, la cruz no sólo tiene implicaciones existenciales [Jungel, E. (1985), Gott als Geheimnis der Welt. Tübingen: Mohr, pp. 280-283], sino que implica una solidaridad de Dios con la historia real, en la que también albergan el dolor, el sufrimiento y la muerte . 
dedicada al problema teologal del hombre: El hombre y Dios (1983). En el segundo libro, El problema filosófico de la historia de las religiones (1993), Zubiri aborda la experiencia de Dios en la historia de las religiones. Y el tercero, El problema teologal del hombre: Cristianismo (1997), tiene por objeto definir la experiencia cristiana, que para él representa la manifestación más alta posible de Dios: la encarnación. En el misterio cristiano, de hecho, Dios se hace hombre y el hombre se convierte en Dios: es la experiencia de la deiformidad y de la deificación.

Para la investigación del problema de Dios, son ineludibles los presupuestos antropológicos y metafísicos, y, especialmente, epistemológicos, que Zubiri expresó, respectivamente, en la original concepción del hombre como una realidad personal y en la «trilogía» dedicada a la inteligencia humana con la concepción original de la inteligencia sentiente ${ }^{3}$. Es a la luz de este itinerario intelectual y existencial como tenemos que entender los trabajos antropológicos y religiosos, que serían ininteligibles si no consideráramos la original teoría metafísica y epistemológica, o sea la «filosofía primera», que implica los siguientes momentos: noético (inteligencia sentiente), noemático (la realidad) y noérgico (la re-ligación).

La persona se realiza en la vida a través de las acciones de las que es actor, agente y autor. Si bien tiene que tener algo que ver «con» las cosas, el hombre en última instancia reside en la realidad; las cosas, por lo tanto, son el vehículo de la misma realidad, que asume el carácter de un fundamento que, en los aspectos de ultimidad, posibilidad e impelencia, muestra una ambivalencia, ya que por un lado se configura como la alteridad radical en relación a mí, y por el otro es lo que permite la realización personal. De ahí aparecen preguntas e inquietudes en torno a este fundamento, que no es causa, sino «dominio», o sea un carácter físico real y, en definitiva, es un poder. El «poder de lo real» se apodera de mí y me domina ${ }^{4}$. Esta es la antítesis del subjetivismo moderno, según el cual el sujeto legisla sobre la realidad. Por el contrario, es la realidad la que se apodera de mí. Esta no es una posición gnoseológica, sino real, de la cual el conocimiento es sólo una consecuencia. El poder de lo real es un apoyo, no tanto y solamente para actuar y vivir, sino, ante todo y sobre todo, para ser real. Entonces, no «vamos a» la realidad, sino que «venimos de» la misma. Este vínculo con el «poder de lo real» es, desde el punto de vista metafísico,

3 A la inteligencia humana, Zubiri ha dedicado, como es bien sabido, una trilogía: ZuBIRI, X. (1980), Inteligencia sentiente. Inteligencia y realidad. Madrid: Alianza; ID. (1982), Inteligencia y logos. Madrid: Alianza e ID. (1983), Inteligencia y razón. Madrid: Alianza. Sobre la antropología, véase ZuBIRI, X. (1986), Sobre el hombre, ed. de Ellacuría, I. Madrid: Alianza. En torno a estos temas, cf. Pintor-Ramos, A. (1994), Realidad y verdad. Las bases de la filosofía de Zubiri. Salamanca: Publicaciones Universidad Pontificia de Salamanca; ID. (1993), Realidad y sentido: desde una inspiración zubiriana. Salamanca: Publicaciones Universidad Pontificia de Salamanca y Savignano, A. (2006), Panorama de la filosofía española del siglo Xx. Granada: Comares.

4 ZubiRI, X. (1993), El problema filosófico de la historia de las religiones. Madrid: Alianza, p. 61 . 
la «religación», cuyo objeto formal no es la naturaleza, sino la persona o, más bien, la naturaleza personal; además cubre la totalidad de la realidad, aunque solamente en la persona humana está actualizada formalmente. La religación es un hecho total y radical ${ }^{5}$. Es ante todo un hecho, como la misma realización personal. Pero se trata de un hecho total, ya que afecta a la realidad personal entera. También es un hecho radical, porque es la re-ligación a la realidad lo que configura a la persona.

Investigando la religión, Zubiri empieza por la re-ligación entendida como análisis de los hechos, distinguiéndose así del método fenomenológico que trata de comprender el sentido de las experiencias religiosas antes de tomar una posición filosófica sobre su verdad o falsedad. Esta perspectiva asume hoy en día un carácter hermenéutico en base a la conocida distinción entre "comprensión» y «explicación», respecto a la cual Zubiri se sitúa en un nivel anterior, es decir, del hecho de la religación al poder de la realidad, bajo la influencia de J. B. Pratt ${ }^{6}$.

La religación, que es la raíz de la realidad y de la realización personal en las diversas formas optativas, no es una obligación moral, o un sentimiento de dependencia incondicional. En la re-ligación, no "vamos hacia» algo que nos perfecciona, sino que «venimos de» algo que previamente nos hace existir. El hombre consiste expresamente en religación o en religión, que, sin embargo, no se basa en la práctica, en la ética, en el sentimiento, no es una "propiedad» que se puede poseer o no, sino que es una dimensión formal de la persona humana, en definitiva, es un fundamento para ser real.

La religación está configurada como una experiencia, un enigma y una manifestación del poder de lo real. La re-ligación es una experiencia que no es simplemente el dato sensible o empírico, sino el acto de comprobar la efectiva realidad de algo. Para la re-legación esta prueba de la realidad consiste en la realización en la persona de las oportunidades encontradas en la realidad. En el hacer «re-ligadamente su propia persona», es decir, realizando las posibilidades, el hombre hace la prueba de lo que es el poder de lo real, hace la prueba de la realidad. Esta prueba es precisamente la inclusión de la ultimidad, de la posibilidad y de la impelencia del poder de lo real en su propia realidad. El hombre, al hacerse realidad personal, pasa por la experiencia del poder de lo real, y por tanto por la experiencia de la misma realidad.

5 Sobre esta teoría original, núcleo de la filosofía de la religión, véase el fundamental ensayo de Zubiri, X. (1935), «En torno al problema de Dios», en ID. (2004 ${ }^{12}$ ), Naturaleza, Historia, Dios. Madrid: Alianza, pp. 361-399. En torno a este tema, que se debe entender a la luz de la evolución intelectual del filósofo español, véase ElLacuría, I. (1964), «La religación, actitud radical del hombre», Asclepio, 16, pp. 97-155; Andrés FernándeZ, J. DE (1966), «Los presupuestos antropológico-metafísicos de la afirmación de Dios en Zubiri", Revista de Filosofía, 26, pp. 125-153 y A. Guy (1964), «La théorie de la religation selon Zubiri», Bulletin Hispanique, 66, pp. 391-395.

6 Véase Pratt, J. B. (1934), The Religious Consciousness. New York: Macmillan, p. 2: "religion is the serious and social attitude of individuals or communities toward the power or powers which they conceive as having ultimate control over their interests and destinies". 
Zubiri introduce un concepto de experiencia, en comparación con el concepto de lo empírico, suficientemente abierto, a través de la creación de esquemas por obra de la razón, como fundamentos de las cosas más allá de nuestra comprensión, por lo que la realidad, en el momento de la aprehensión, es el objeto de la intelección a la luz del fundamento esbozado ${ }^{7}$.

¿Se puede decir lo mismo en torno a la experiencia religiosa? Para Zubiri, la religión no es una visión del mundo, ni una explicación de la realidad en su totalidad, sino que es la plasmación de la religación. El fundamento es también la base de la realidad, a condición de que la realidad no se entienda como la totalidad hegeliana, sino como una formalidad constitutivamente abierta. Dios, para Zubiri, no se experimenta principalmente como verdad sobre la totalidad, sino como el fundamento de la religación. En consecuencia, construimos nuestra vida vinculados a este fundamento.

¿Pero podemos tener una experiencia concreta — sin calificarla como una teoría de la totalidad - de este fundamento de la religación? Según Zubiri, el hombre no puede normalmente experimentar a Dios, pero puede ser una experiencia de Dios, viviendo su vida atado a lo que hemos postulado como el fundamento último del poder de lo real. ¿Esta es realmente una prueba o simplemente una experiencia de nuestra fe en Dios? Para Zubiri, Dios no se postula solamente como algo más allá de nosotros, sino como el más cercano, ya que no es trascendente a las cosas, sino que está en las cosas. Basando nuestra vida en Dios, entendemos nuestra realidad como una experiencia de Dios.

La religación es también una manifestación del poder de lo real, que aparece en la actualización de las notas o elementos de la realidad. De este modo, lo que se manifiesta es el mismo poder de la realidad como re-ligante. La religación es también enigmática. La raíz de este enigma se encuentra en el hecho de que cuando estamos en las cosas reales estamos en la realidad. El momento de la realidad en la cosa real es más que la cosa real y, además, el hecho mismo de estar entre las cosas reales nos obliga a estar en la realidad, que es más que las cosas reales. De hecho, las cosas reales son como el vehículo del poder de lo real, al que estamos religados, y por eso es por lo que, al quedarnos con las cosas, estamos en la realidad.

El poder de lo real es el fundamento de la re-ligación personal. Esto no quiere decir que en un momento dado el hombre caiga bajo la influencia del poder de lo real. El dominio del poder de lo real sobre el hombre es constitutivo e intrínseco: es como un soporte para ser real, lo que hace que sea él mismo. El hombre es, de hecho, una realidad personal, ya que depende del poder de lo real, cuyo dominio nos fija en la realidad. Esta fijación no se opera dirigiéndonos hacia la realidad, sino procediendo de ella. La inserción en la realidad es paradójica. Por un lado, el poder de lo real nos fija y une a la realidad; por otro lado, nos constituye como "absolutos», o sea distintos respecto a esta. Paradójicamente, el dominio del poder de la realidad nos une y al mismo tiempo nos hace

7 En torno al concepto de experiencia, cf. ZuBIRI, X., Inteligencia y razón, pp. 222-257 y El hombre y Dios, pp. 307-317. 
relativamente absolutos. Este vínculo especial es la re-ligación. A través de ella estamos obligados por la fuerza de la realidad, estamos bajo su dominio. Siendo re-ligado, el hombre tiene el apoyo suficiente para ser relativamente absoluto.

El poder de lo real se da en cada una de las aprehensiones de la realidad, por lo tanto, no se trata sólo del contexto religioso, con referencia a lo sagrado, lo numinoso, lo fascinante, lo misterioso, lo terrible ${ }^{8}$. Se trata de una definición de la religión a la luz de la re-ligación como hecho universal anterior a cualquier sentido religioso (lo sagrado, lo numinoso, etc.) en que insiste el enfoque fenomenológico. Los múltiples sentidos religiosos son la plasmación concreta e histórica de la religación en las diversas religiones.

Gracias a la religación estamos, por lo tanto, empujados físicamente hacia la realidad, que se apodera de nosotros. El poder de lo real es enigmático, y este enigma da carácter a mi logro personal: es la naturaleza problemática del fundamento. Esto se muestra: 1) en la inquietud, no sólo en el sentido de la famosa afirmación agustiniana de la búsqueda de la felicidad en el campo psico-antropológico, sino, sobre todo, en el nivel metafísico; se trata, de hecho, de la inquietud que se produce por la misteriosidad del poder de lo real; aunque con frecuencia puede ser experimentada como ansiedad y preocupación, sin embargo, generalmente afecta a la realización esencialmente personal. Además, 2) en la voz de la conciencia, que no es comparable con el imperativo categórico de Kant, ni tampoco es un momento psicológico, sino metafísico; es una llamada de la realidad misma, que, a través de la inteligencia sentiente, toma la apariencia de una noticia. Por este lado, la voz de la conciencia es la llamada de la realidad. En fin, 3) en la volición sentiente mediante la cual es posible distinguir entre la realidad-objeto y la realidad-fundamento, ya que se elige esencialmente para la realización personal sólo esta última opción. La volición sentiente, que no es comparable con la nietzscheana Wille zur Wahrheit, se actualiza en la inteligencia sentiente y llega a la verdad real.

La voluntad de verdad es la búsqueda, a través de la inteligencia sentiente, de la articulación en todas las cosas de «su realidad» con «la realidad». Esta investigación fundamental y fundacional de mi propia realidad «dentro y hacia» la "realidad-fundamento» se puede definir, con Bergson, como experiencia metafísica, una experiencia que está en busca del fundamento del poder de lo real; experiencia que con más precisión Zubiri llama experiencia teologal, muy diferente de la experiencia teológica9 .

8 Otтo, R. (1917), Das Heilige. Breslau: Trewendt \& Granier; M. Eliade (1949), Traité d'histoire des religions. Paris: Payot. Para una crítica de esta perspectiva, véase ZuBIRI, X., El problema filosófico de la historia de las religiones, pp. 18-28.

9 En torno a este problema, es fundamental, además del libro El hombre y Dios, la tercera parte de Naturaleza, Historia, Dios. Sobre este aspecto, cf. Savignano, A. (1996), «La dimensione teologale dell'uomo e la teologia fondamentale», en A. BABOLIN (ed.) (1996), Teologia filosofica e filosofia della religione. Perugia: Benucci, pp. 339-373. Cf. López Quintás, A. (1984), "La dimensión teologal del hombre según Zubiri», en Babolin, A. (ed.) (1984), Antropologie tipologiche e filosofia della religione. Perugia: Benucci, pp. 245-283. También sobre este tema, es fundamental tener en cuenta la evolución intelectual del filósofo español. 
Si es cierto que el hombre, para darse cuenta de sí mismo como persona, no puede evitar la experiencia teologal, entonces la naturaleza problemática de la realidad-fundamento es formalmente el problema de Dios. Este no es el lugar para analizar a fondo este tema, sino tan sólo para subrayar que, antes de este famoso libro sobre el hombre y Dios, Zubiri había tratado de esbozar, a través de la re-ligación, el marco conceptual y el contexto interpretativo adecuado en el que el problema de Dios fuera inteligible para el hombre moderno que, a diferencia de la época medieval, ha puesto en duda la posibilidad misma de su abertura a la trascendencia. Zubiri de esta forma muestra una conciencia tan aguda que la cuestión de la inteligibilidad de Dios es anterior a la demostración de su existencia, al menos para el mundo contemporáneo. La justificación intelectual no es de naturaleza psicológica, ética, social, sino que se basa en la re-ligación, ya que es un camino real. El vínculo «re-ligante» revela la raíz de la fundamentalidad de la existencia, ya que muestra que existe lo que «re-liga»: la deitas, que se nos muestra como simple correlato de la re-ligación: estamos fundados en la re-ligación que a su vez se funda en la deidad ${ }^{10}$. Sin embargo, con el descubrimiento de la deidad, estamos en la primera etapa de la ruta de acceso real a Dios, si bien los otros momentos - la realidad divina y Dios mismo - se basan el uno en el otro. La deidad es como un enigma que impulsa inexorablemente a proceder. Este segundo paso, más demostrativo en sentido estricto, conduce a la realidad divina, que es causa absoluta y trascendente y separada del mundo. El descubrimiento de la realidad divina conduce a una nueva etapa que implica la cuestión de quién es esta causa: se trata de Dios, como inteligencia, voluntad y donación: los mismos rasgos que caracterizan a la posición teísta ${ }^{11}$.

Hasta ahora, el problema de Dios se analizó quoad nos y, por decirlo así, a parte objecti; por eso es esencial examinarlo a parte subjecti. De aquí, el verdadero viaje comienza desde la re-ligación al poder de lo real hasta su fundamento, el acceso al cual, siendo libre, implica una justificación intelectual. Este encuentro se realiza en el ámbito del poder de lo real, porque las cosas, sin necesidad de identificarse con Dios (panteísmo), ni siendo una emanación de Dios, sin embargo son reales sólo en Dios, es decir, son Dios ad extra gracias a la creatio ex nihilo. Aprovechando el poder de lo real, el hombre hace la experiencia fundamental de que Dios es trascendente en las cosas y el ser humano es de alguna manera Dios: es Dios en términos humanos, es decir, la realidad relativamente absoluta que es formalmente experiencia de la realidad absolutamente absoluta. Sin que deje de ser abismal la diferencia ontológica entre las dos realidades, el hombre, en la experiencia fundamental, incluye a Dios hasta conformarse a él. Esta forma de posesión será, además de una verdadera intelección, una realización experimental de nuestra realidad humana en Dios. Esta forma de posesión, además de ser real, constituye una realización experimental de su propia realidad humana en Dios.

10 Zubiri, X., «Introducción al problema de Dios», en Naturaleza, Historia, Dios, p. 357.

11 ZuBiri, X. (1942), «Nuestra situación intelectual», en Naturaleza, Historia, Dios, p. 31. 
Era necesario subrayar todo esto, a fin de disipar los malentendidos sobre todo en torno a la relación entre la antropología y la teología, el papel de la demostración en el problema de Dios, la cuestión decisiva del ateísmo, la aclaración del verdadero camino hacia Dios; también era necesario expresar, con lenguaje y perspectiva metafísicos, tesis defendidas desde un punto de vista ontológico; finalmente surgió la necesidad de dar una forma orgánica y sistemática a estas ideas: son estas algunas de las preguntas que el libro póstumo, El hombre y Dios, lamentablemente hasta ahora incompleto, ayuda a clarificar y hasta resolver ${ }^{12}$. Como es sabido, existe una nueva edición crítica de este libro que permite resolver muchas cuestiones y problemas.

La relación entre el hombre y Dios no es abstracta sino concreta, de modo que el hombre es experiencia de Dios y viceversa. Dada esta configuración, se puede decir que el camino de la re-ligación — cuya esencia es la tensión teologal— la trayectoria intelectual y la experiencia de Dios constituyen la dimensión teologal del hombre. De esta forma se eliminan muchas de las insinuaciones y dudas sobre la relación entre la antropología y la teología, que se derivan del hecho de que el ensayo sobre el problema teologal del hombre (1975) se incluyó en un libro dedicado a Karl Rahner. Desde entonces, sin embargo, Zubiri confirmó la diferencia entre la investigación teológica — que es esencialmente teocéntrica- y la dimensión teologal; por otra parte, aunque se trata de una dimensión humana, es precisamente una dimensión por la que el hombre se funda en el poder de lo real. Eso es todo lo contrario de la antropología, ya que se trata de la inserción del hombre en la realidad. La unidad intrínseca y formal que se expresa en la religación al poder de lo real, en la voluntad de verdad, en la experiencia de la búsqueda de la realidad personal absoluta, constituye la dimensión teologal del hombre.

El problema de Dios conduce a una revolución de los esquemas conceptuales comunes no sólo desde un punto de vista antropológico-epistemológico, sino sobre todo a nivel metafísico. El hombre no llega a Dios por una facultad especial u órgano, ni tampoco por el sentimiento, porque esto implicaría deslizarse hacia actitudes psicologistas y subjetivistas; no lo hace por el mero conocimiento intelectual, porque es abstracto; ni por la acción o la práctica, ya que Dios no es un incentivo para actuar, sino un fundamento para ser. No se trata, por tanto, de un método de inmanencia (Blondel) o de trascendencia, porque lo que es Dios en el hombre es re-ligación. De ahí la tarea de la misma teología fundamental, que ya no debería investigar los así llamados praeambula fidei, sino centrarse en el tema de la inteligibilidad y del sentido del problema de Dios.

La filosofía de la religión no es, por tanto, solamente un estudio psicológico e histórico-social de la experiencia religiosa; así como no se encuentra sólo en un ámbito intelectual o emocional; sin embargo, sin descuidar estos aspectos, Zubiri subrayó el carácter ontológico (después dirá: metafísico) de la religión. La filosofía de la religión se diferencia incluso de la apologética, del estudio

12 Sobre este libro, remitimos a nuestra introducción a la nueva edición de ZuBIRI, X. (2013), L'uomo e Dio. Bari: Pagina. 
comparativo de la historia de las religiones, ya que se limita a una consideración formal de la religión desde el punto de vista metafísico, a fin de delimitar las categorías de esa forma peculiar e irreductible de vida. La misión de la filosofía de la religión es el análisis de la religión como una forma de ser y de la realidad humana en la dimensión personal, social e histórica, para resolver la cuestión crucial de la verdad de la religión en el sentido de determinar las condiciones bajo las cuales la religión es vera religio ${ }^{13}$.

Los múltiples modos y dimensiones de la experiencia humana en el nivel individual, social e histórico constituyen la historia de las religiones, que es la experiencia teologal de la humanidad individual, social e histórica, en orden a la verdad última del poder de lo real: Dios ${ }^{14}$. El problema filosófico de la historia de las religiones - que, por supuesto, se distingue del problema teológico- es el de examinar el impulso de la divinidad en el espíritu humano. La religión, de hecho, es la plasmación de la religación, porque a fin de cuentas siempre asume el carácter de una concepción de Dios, del mundo y del hombre. La religión tiene, en primer lugar, un rasgo diferente que depende de las diferentes ideas en torno a Dios: la visión politeísta, panteísta y monoteísta. A cada uno de estos tres conceptos, que es verdadero a su manera, el hombre llegó por distintas rutas. La diversidad de estas vías y estas ideas depende de la difracción de la realidad de Dios en el espíritu humano. Las religiones no sólo son diferentes, sino que también son históricas, porque cada una de ellas implica diferentes posibilidades de la inteligencia de esa divinidad. La historia no es un sistema de realidades, sino un sistema de posibilidades ${ }^{15}$. Estas tres ideas de Dios son el resultado de tres itinerarios: el camino de la dispersión, que conduce al politeísmo; el camino de la inmanencia, que conduce al panteísmo; el camino de la trascendencia, que llega a la realidad de Dios. Si bien la diversidad de ideas representa el resultado de la difracción de la presencia de Dios en el espíritu humano, la diversidad de los itinerarios es justamente el camino que el espíritu humano necesita para llegar del Dios que lleva dentro de sí al Dios real y genuino.

Entre las religiones históricas, Zubiri muestra la excelencia del cristianismo, que es la verdad radical y formal de todas las religiones, ya que es la experiencia teologal suprema, y que no hay mayor forma de ser humanamente Dios que la deiformitas. En una época como la nuestra, caracterizada por el inevitable diálogo ecuménico, el filósofo español señala que el cristianismo es la forma más radical entre las diferentes formas de religión, ya que implica una trascendencia no solo histórica, sino teologal ${ }^{16}$.

13 Cf. Zubiri, X. (1937), «Note sur la philosophie de la religión", Bulletin de l'Institut Catholique de Paris, 18, pp. 334-341.

14 ZubiRi, X., El hombre y Dios, p. 380.

15 En torno al concepto de historia, véase A. SAvignano (2010), «Concepción y papel de la historia en Heidegger y Zubiri", en Ocho miradas al pensamiento español del siglo XX. Pamplona: Ediciones de la Universidad de Navarra, pp. 105-121.i

16 Cf. Zubiri, X. (2014), Il problema filosofico della storia delle religioni, ed. de A. SAVIGnANo. Brescia: Morcelliana. 
No hay duda de que la reflexión de Zubiri cobra características originales cuando se la considera a la luz de una filosofía de la religión, que difiere tanto de la fenomenología religiosa como del enfoque analítico de la religión. La perspectiva zubiriana hace que sea posible mantener un grado de neutralidad hermenéutica respecto a la fenomenología de la religión, donde se subraya con razón la necesidad de una sensibilidad religiosa o del sentimiento de pertenencia a una tradición en la que esa experiencia ha sido vivida - a priori religioso (R. Otto) o pre-comprensión (Gadamer). Zubiri, aunque juzga esencial todo esto, se sitúa en un nivel anterior y más fundamental que el sentido, ya que la religación no se aplica, en primer lugar, al significado, sino que es una dimensión accesible a un análisis de la realidad actualizada en la aprehensión primordial. El sentido no es objeto de la aprehensión primordial de la realidad, sino del logos ${ }^{17}$.

Respecto a la filosofía analítica de la religión, Zubiri nota que la religión no es principalmente una cuestión de significado, ya que es radicalmente religación. Pero esto no implica una negación de la función del lenguaje religioso. En este sentido, puesto que la religación no es solo un hecho, realizado en la aprehensión, sino un hecho positivo, que cualquier persona puede aprehender $^{18}$, implica la atribución de significado a las expresiones religiosas, a condición de entenderlo (el significado), no en términos de representación sino de «direccionalidad». Las expresiones religiosas, lejos de ser meras expresiones emocionales, tienen un significado coherente con la intención de dar sentido precisamente a la religación ${ }^{19}$. Zubiri analiza, antes de los significados religiosos, la re-legación como algo que aparece en nuestras acciones.

Esto no significa evadir el problema crucial de la verificabilidad de las expresiones religiosas. Como es bien sabido, los filósofos analíticos en general han revelado la dificultad de verificar las afirmaciones teístas ${ }^{20}$. En cambio, la filosofía zubiriana permite enfatizar la diferencia entre las proposiciones acerca de lo que se realiza en la aprehensión y las proposiciones — por ejemplo las monoteístasque se refieren a quien, por definición, trasciende todos los actos ${ }^{21}$.

Università di Trieste

Armando Savignano

armando.savignano@tin.it

[Artículo aprobado para publicación en este número extraordinario en noviembre de 2014]

17 Cf. Zubiri, X., Inteligencia y logos, pp. 89-107.

18 Cf. ZuBiri, X., Inteligencia y razón, pp. 180-186.

19 Sobre el papel del lenguaje en Zubiri, véase Pintor-Ramos, A., Realidad y verdad. Las bases de la filosofía de Zubiri, pp. 185-239.

20 Cf. Flew, A. (19665), New Essays in Philosophical Theology. London: Macmillan.

21 En torno a la filosofía de la religión de Zubiri, véase Gracia, D. (1994), «Religación y religión en Zubiri», en Fraijo, M. (ed.) (1994), Filosofía de la religión. Madrid: Trotta, pp. 491512; Pintor-Ramos, A. (2001), «Religación y prueba de Dios en Zubiri», Razón y Fe, 218, pp. 319-336; Solari, E. (2001), «La filosofía de la religión de Zubiri», Revista Agustiniana, 42, pp. 517-635 y Millas, J. M. (2004), La realidad de Dios. Su justificación y sentido en X. Zubiri y J. Monserrat. Roma-Madrid: E.P.U.G-UPC. 